\title{
The golden anniversary of the Austrian Society of Hematology and Medical Oncology (OeGHO)
}

\author{
Wolfgang Hilbe
}

Received: 21 December 2019 / Accepted: 21 December 2019 (C) Springer-Verlag GmbH Austria, part of Springer Nature 2020

In 1970 the OeGHO (Austrian Society of Hematology and Medical Oncology) was established by a pioneering group of dedicated hematologists around Professor Fleischhacker (1970-1975), a well-known expert from Vienna. In its initial years the society was called Austrian Society of Hematology. The primary goal of the society was to generate a platform of scientific communication and exchange between experts. The members interacted together and constantly updated the group on their various scientific activities. According to international developments the focus of the OeGHO was extended to solid cancers. Consequently, in 1977 under the guidance of Professor Stacher (1975-1981) the OeGHO was renamed to be the Austrian Society of Hematology and Oncology. The OeGHO turned out to be a meeting point and offered young colleagues the chance to present their scientific activities, to gain their first experiences in presenting scientific data and communicate with peers. Additionally, OeGHO was the main driver to introduce an officially approved specialization of "Hematology and Medical Oncology" in 1994 within the subject of internal medicine. Under the presidency of Professor Gastl (2008-2013) a more professional and modern organization was implemented. An office was founded and a managing director was employed. In those years the role of medical oncologists was heavily questioned by the organ specialists. Therefore, we were forced to stand up and plead for the special role of our field of expertise within the interdisciplinary management of cancer patients. In those days beside the traditional scientific focus our society developed to be an independent provider of professional medical education which led to the foundation of the "Onconovum academy". When Professor Samonigg (2013-2017) took the lead, he realized that our society bears the responsibility to communicate a well-defined position on health care topics with the public. He initiated a famous media campaign to fight against smoking, called “don't smoke”. The campaign succeeded and the political parties prohibited public smoking in 2019, as it was already standard in other European countries. Furthermore basic and clinical cancer research has seen great advances in 50 years.

Fifty years after foundation of the OeGHO we are proud to be a very active society having about 800 full members. At our national congress up to 800 participants are registered and more than 100 experts play an active role in scientific sessions. In 2020, supporting scientific activities, offering highly qualified education and communicating relevant heath care topic are our three major fields of activity. At the end we feel responsible that young doctors become an expert, that new scientific innovations could enter the clinical path, that patients can be managed according to a present "state of the art". Furthermore our aim is to support the shift in the culture of cancer research, moving towards multidisciplinary, integrated, and patient-centered work as the ultimate goal of our society. Our members and their engagement in multiple fields in hematology and medical oncology are OeGHO. Thus, we are OEGHO and the future of OEGHO lies in our hands and we look forward to the next 50 years.

\footnotetext{
Univ.-Prof. Dr. W. Hilbe $(\bowtie)$

Austrian Society of Hematology and Medical Oncology (OeGHO), Vienna, Austria

Magazine of European Medical Oncology, Vienna, Austria wolfgang.hilbe@meduniwien.ac.at
} 


\section{editorial}

\section{Chairmen of OeGHO}

- 1970-1975: Univ.-Prof. Dr. Hanns Fleischhacker

- 1975-1981: Univ.-Prof. Dr. Alois Stacher

- 1981-1985: Univ.-Prof. Dr. Alois Wagner

- 1985-1991: Univ.-Prof. Dr. Heinz Huber

- 1991-2000: Univ.-Prof. Dr. Klaus Lechner

- 2000-2003: Univ.-Prof. Dr. Heinz Ludwig

- 2003-2008: Univ.-Prof. Dr. Dieter Lutz

- 2008-2013: Univ.-Prof. Dr. Günther Gastl

- 2013-2017: Univ.-Prof. Dr. Hellmut Samonigg

- 2017-2019: Univ.-Prof. Dr. Andreas Petzer

- 2019-ongoing: Univ.-Prof. Dr. Wolfgang Hilbe

Conflict of interest W. Hilbe declares that he has no competing interests.

Publisher's Note Springer Nature remains neutral with regard to jurisdictional claims in published maps and institutional affiliations.

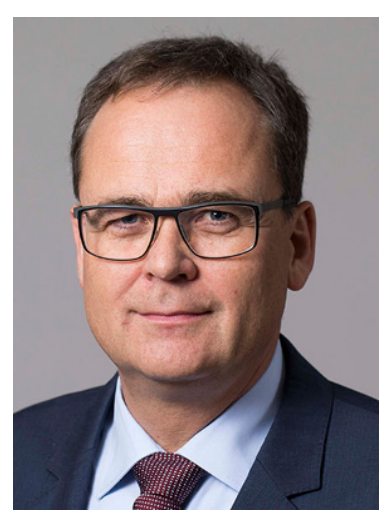

Wolfgang Hilbe

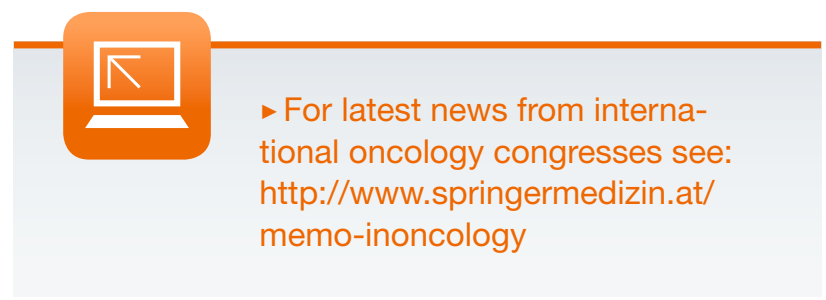

